\title{
'Petrol from plants' claim baffles Indian scientists
}

New Delhi. Indian scientists have been baffled by the claims of a 30-year-old selftaught chemist from the southern state of Tamilnadu that he is able to power a scooter using 'petrol' produced by adding leaves and bark extracts from a native herb to tap water.

Ramar Pillai demonstrated his backyard experiments to a scientific audience last week in the chemistry laboratory of the Indian Institute of Technology (IIT) in New Delhi. Scientists who watched the water turn into an yellowish, inflammable liquid admit that they have no explanation at present.

But they remain convinced by the demonstration. "It is just unbelievable but true," says N. K. Jha, head of the chemistry department. Valangiman Ramamurti, the secretary of the Department of Science and Technology (DST), who himself performed the experiment said: "Like everyone else I was sceptical, but we now know it is not an Indian rope trick."

Pillai's curiosity had initially been stimulated as a schoolboy when a spark from a camp-stove ignited the leaves of a distant plant during a school picnic. Ten years later, he tracked down the 'burning plant' and started experimenting with it, using knowledge picked up from chemistry textbooks.

The process he developed to make the fuel involves cooking the leaves of this plant — although other parts will do - for about ten minutes in hot water to which a little salt and a few drops of lemon juice or citric acid have been added. As the liquid cools, certain chemicals - believed to be catalysts - are added, and the contents allowed to settle.

Because it is lighter than water, the herbal fuel rises to the top, and is filtered off. Pillai says his fuel would cost one rupee (3 US cents) a litre. Commercial production should not be a problem, because the plants involved grow like tea bushes, with leaves continuing to grow to replace those that have been plucked.

Last week's experiment started with 55 grams of the herbal leaves and bark, and 1,000 millilitres of tap water. Just over half an hour later, this had produced 460 millilitres of a liquid that burned and smelled like kerosene, and on distillation yielded a fraction boiling at $170^{\circ} \mathrm{C}$ ).

According to the National Chemical Laboratory in Pune, analysis of the fuel has shown it to be a pure hydrocarbon, although no details have been published for fear of prejudicing patent applications. Tests at the mechanical engineering department of IIT Madras found the fuel to be "better than [real] petrol" in terms of economy. Furthermore, being of plant origin, it contains no sulphur and the resulting exhaust is therefore cleaner.

"We have no doubt that we are sitting on something very big, but we want to proceed cautiously and systematically," says Ramamurti. Officials at the DST strongly believe that the herbal process, if commercialized, could have a major impact on the Indian economy.

The DST has already agreed to provide funds for a pilot plant at Rajapalayam, capable of producing 300 litres a day. At the same time, India's leading chemists will try to understand how the process works, as

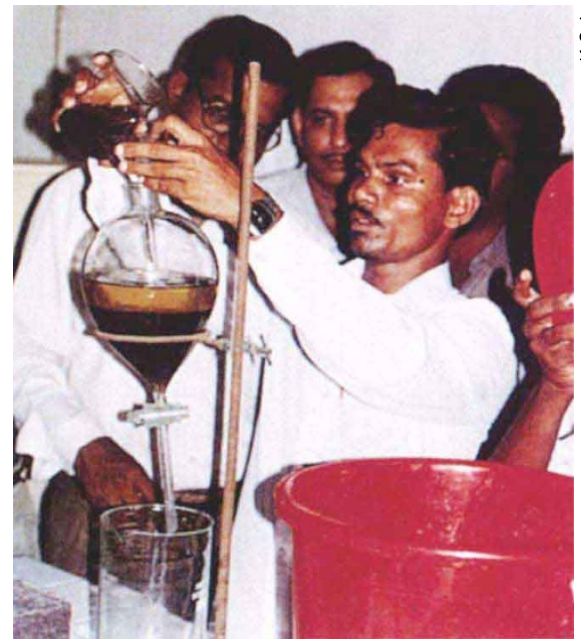

Liquid gold? Pillai produces his 'fuel' using leaves of a native herb and water.

plans are drawn up for a production capacity of one million litres a day. According to Ramamurti, all of India's main scientific agencies will be provided with the necessary funding to participate.

Scientists who have watched the experiment accept that the process generates a hydrocarbon fuel. But they describe this as a 'miracle' in the absence of accepted experimental protocols, and lack of details of the herb and catalysts used.

Two factors in particular remain baffling. "The process is too fast," says Ratna Choudhury, a chemist at IIT who has been studying methods of extracting hydrocarbons from the so-called 'petro-crops' such as jojoba. Second, although the hydrogen could, in principle, be provided by the water, they have no explanation for the presence of large quantities of carbon; a suggestion that it might be 'sucked in' from the air is widely considered to be implausible.

Pillai says that he will disclose the secrets only after his invention is patented. DST hopes that the patenting process will be completed within two months, at which point controlled experiments will be able to start.

K. S. Jayaraman

\section{Foundations funding biomedical bodies 'should shift focus'}

San Francisco. Private foundations should play a greater role in helping academic biomedical institutions adjust to the tightening of federal funds, increasing financial pressures on medical centres, and the growing role of industry in research, according to the University of California's San Francisco Center for the Health Professions. A report prepared jointly with the Pew Biomedical Scholars Program warns that foundations are exacerbating a mismatch between training and job opportunities by continuing to emphasize doctoral training and fellowships.

Carolyn Asbury, director of the health and human services programme of the Pew Charitable Trusts, says that foundations should instead be trying to meet industry's demand for more interdisciplinary training, to focus on policy issued, and to develop centres where investigators from different backgrounds can share resources.

The report predicts that the 50-per-cent increase in doctoral awards in the biological sciences over the past $\mathbf{1 0}$ years will lead to an oversupply of biomedical researchers.

Funding tends to support established laboratories as research becomes more costly and the emphasis on practical applications grows. This further harms the prospects for promising young scientists, says Edward O'Neil, co-director of the Center for the Health Professions and principal author of the report.

Interviews with 200 Pew scholars indicate that junior-level faculty members are growing disenchanted, with good scientists leaving for business, law and other fields. The scientists interviewed by O'Neil were worried they might not be able to continue in science, even though they had received one of the most sought-after young faculty awards in the United States.

Federal spending on biomedical research has not kept pace with increased costs, the report points out, in spite of a 6.5-per-cent increase in federal funding for the National Institutes of Health (NIH) during the 1996-97 budget cycle. In 1992, industry started to become the most important source of money for biomedical research. In 1994 , industry spent $\$ 17.1$ billion on such research, compared to $\$ 14.5$ billion from the federal government.

O'Neil says NIH grants and the reward systems at universities must change to encourage smaller laboratories to flourish. Large laboratories should develop a new class of mid-level senior scientists, instead of just a principal investigator plus fellows and graduate students. Universities should seek ways to fund a scientific infrastructure independent of grants.
Sally Lehrman 